\title{
Ferromagnetic resonance force microscopy on microscopic cobalt single layer films
}

\author{
Z. Zhang ${ }^{\text {a) }}$ and P. C. Hammel ${ }^{\text {b) }}$ \\ Los Alamos National Laboratory, Los Alamos, New Mexico 87545 \\ M. Midzor and M. L. Roukes \\ California Institute of Technology, Pasadena, California 91125
}

J. R. Childress ${ }^{\mathrm{c})}$

University of Florida, Gainesville, Florida 32611

(Received 24 February 1998; accepted for publication 31 July 1998)

\begin{abstract}
We report mechanical detection of ferromagnetic resonance (FMR) signals from microscopic Co single layer thin films using a magnetic resonance force microscope (MRFM). Variations in the magnetic anisotropy field and the inhomogeneity of were clearly observed in the FMR spectra of microscopic Co thin films 500 and $1000 \AA$ thick and $\sim 40 \times 200 \mu \mathrm{m}^{2}$ in lateral extent. This demonstrates the important potential that MRFM detection of FMR holds for microscopic characterization of spatial distribution of magnetic properties in magnetic layered materials and devices. (c) 1998 American Institute of Physics. [S0003-6951(98)01640-4]
\end{abstract}

Ferromagnetic resonance (FMR) is an important tool for characterizing magnetic materials. ${ }^{1,2}$ It has played a particularly important role in studies of the magnetic multilayer systems which are becoming widely used in the recording industry as recording read heads and/or media where it has been used to measure the dependence of the interlayer exchange coupling on the thickness of the spacer layer. The ability to perform microscopic FMR would be extremely valuable, enabling characterization, on a microscopic scale, of distributions of magnetic anisotropy and exchange energies in magnetic devices. Microscopic FMR cannot be performed using conventional techniques for two reasons. First, the sensitivity of conventional FMR is inadequate; for most magnetic thin films, such as $\mathrm{Co}$ and $\mathrm{Fe}$, sample areas on the order of $(\mathrm{mm})^{2}$ are needed in order to obtain sufficient signal at $X$ band. Second, conventional FMR is performed in a uniform magnetic field so there is no means to identify the spatial origin of a particular contribution to the FMR signal.

We have recently demonstrated the feasibility of microscopic FMR ${ }^{3}$ by means of the highly sensitive magnetic resonance force microscope (MRFM) ${ }^{4,5}$ The MRFM mechanically detects the resonance signal by sensitively detecting the oscillatory response of a micromechanical resonator. ${ }^{6,7}$ Even before optimizing the instrument, the excellent force sensitivity of the MRFM has enabled detection of ferromagnetic resonance signals from magnetic thin films only tens of microns in lateral extent. ${ }^{3}$ As in magnetic resonance imaging, the MRFM experiment is performed in a strong field gradient; this allows the MRFM to confine the excitation of spin precession to a well-defined surface of constant magnetic field (or "sensitive slice") where the magnetic resonance condition is met. Spatially resolved microscopy is achieved

\footnotetext{
${ }^{a)}$ Present address: Seagate Technology, Mailstop 5-601, 3061 Zanker Road, San Jose, CA 95134.

${ }^{\text {b) }}$ Author to whom correspondence should be addressed. Electronic mail: pch@lanl.gov

c) Present address: IBM Almaden Research Center, San Jose, CA 95120.
}

by scanning this sensitive slice through the sample., ${ }^{8,9}$

In our previous demonstration of microscopic FMR using the MRFM, a single crystal yttrium iron garnet (YIG) thin film was used. ${ }^{3}$ YIG was chosen because it has very strong FMR intensity and narrow resonance linewidth $(<1 \mathrm{G})$. However, typical magnetic devices are composed of metallic ferromagnets such as $\mathrm{Co}$ and $\mathrm{Fe}$. These have much larger FMR linewidths (of order $100 \mathrm{G}$ ), and hence, weaker signals, making the signals harder to detect. It is also essential that the resonant field be substantially larger than the linewidth, hence, that the irradiation frequency exceed $\sim 2-3 \mathrm{GHz}$. Here, we report successful detection of the FMR signal from a microscopic, single layer Co thin film by means of a MRFM instrument. Irradiation at high frequency $(\sim 8 \mathrm{GHz}$, here) and implementation of a MRFM geometry, the perpendicular geometry, as described immediately below, were essential features of the experiment.

The MRFM apparatus used in this work has been described earlier in Refs. 3 and 9. The polycrystalline Co films were deposited onto the tip of the single crystal Si cantilever over polycrystalline $\mathrm{Ag}$ underlayers; the films were capped with protective Ag films. The sizes and locations of the films were determined by a simple mask. Results from three samples will be presented. Sample 1, $1000 \AA$ thick, was sputter deposited at a substrate temperature $<50{ }^{\circ} \mathrm{C}$ onto a $30 \AA$ Ag layer. Samples $2(1000 \AA)$ and $3(500 \AA)$ were thermally evaporated onto $50 \AA \mathrm{Ag}$ layers. $\mathrm{A} \mathrm{Nd}_{2} \mathrm{Fe}_{14} \mathrm{~B}$ bar magnet ( $\sim 6.4 \mathrm{~mm}$ in diameter and $\sim 6.4 \mathrm{~mm}$ long) was used to generate the field gradient $\nabla \mathbf{B}$, and therefore, a force $\mathbf{F}$ on the cantilever:

$$
F_{x}=m_{x} \frac{\partial B_{x}^{\mathrm{bar}}}{\partial x}+m_{z} \frac{\partial B_{x}^{\mathrm{bar}}}{\partial z}
$$

where the oscillatory displacement of the cantilever is parallel to the $x$ axis, and the axis of the bar magnet is parallel to the $z$ axis. The components of the magnetic moment of the Co film along these axes are represented by $m_{x}$ and $m_{z}$. 


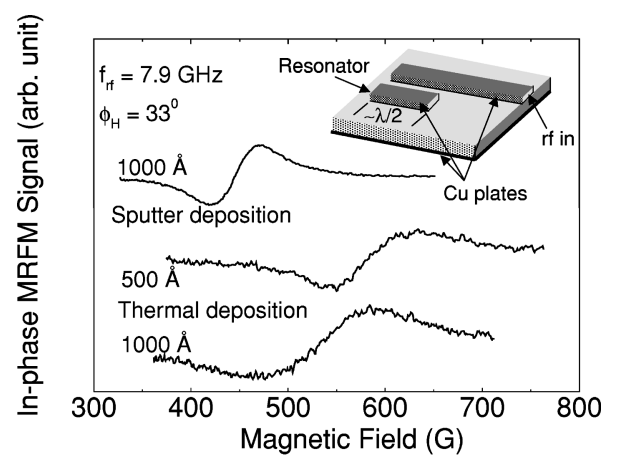

FIG. 1. MRFM spectra of three single layer Co thin films using transverse geometry MRFM. The experiment was performed in air and at room temperature using anharmonic modulation. The angle between the external field and the film plane is $\phi_{H} \sim 33^{\circ}$. The inset shows the design of the microstrip resonator.

For these measurements the earlier apparatus was modified in two respects: we employed a geometry in which the axis of the bar magnet is oriented perpendicular to the motion of the cantilever, in contrast to the conventional MRFM geometry where these are parallel, and we used microstrip resonator to generate the rf field (see inset, Fig. 1). The geometry, depicted in the inset to Fig. 2, was selected because the applied field is in the film plane enabling us to saturate the film with a modest magnetic field (of order hundreds of gauss). In contrast, for the parallel geometry, the magnetic field would be applied perpendicular to the film plane and $H_{\text {res }} \sim 20 \mathrm{kG}$ would be required in order to achieve the FMR condition at the $X$ band: $H_{\text {res }}=4 \pi M_{s}+\omega / \gamma$, where $\omega$ is the rf angular frequency, $\gamma$ is the gyromagnetic ratio and $M_{s}$ is the saturation magnetization of the film. The perpendicular geometry has disadvantages, however. First, the force in the perpendicular geometry is directly related to the field gradient $\partial B_{x}^{\mathrm{bar}} / \partial z$ assuming that the magnetic moment of the film stays close to the film plane. This reduces the sensitivity from that obtained in the parallel geometry where the force is proportional to a much larger gradient $\partial B_{z}^{\mathrm{bar}} / \partial z$. Second, in order to obtain a finite value of $\partial B_{x}^{\mathrm{bar}} / \partial z$, the film must be placed off the axis of the bar magnet. As a result, the external field is applied at an angle $\phi_{H}$ with respect to the film plane. The FMR resonance field of a magnetic film varies with $\phi_{H}$

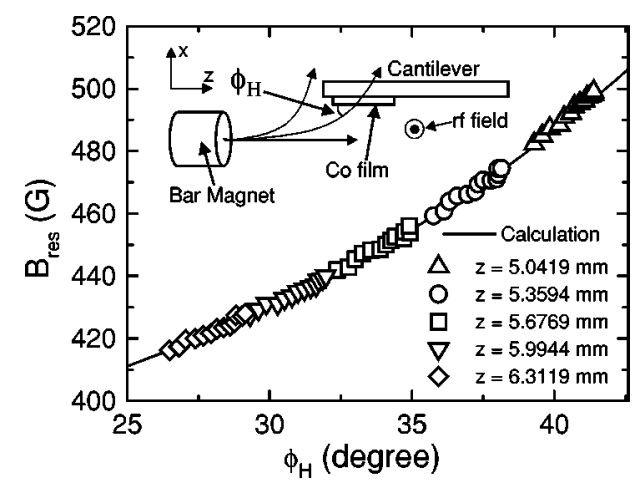

FIG. 2. Dependence of the resonance field on the angle $\phi_{H}$ between the applied magnetic field and the film plane for the sputtered sample. The solid line is a theoretical fit from a classical FMR theory with $g=2.18$ and $4 \pi M_{\text {eff }}=17.6 \mathrm{kG}$. A vanishing crystalline anisotropy field was assumed in the calculation. The inset shows a schematic diagram of the MRFM apparatus. method and film thickness. The magnetic anisotropy of the
Downloaded 25 Feb 2006 to 131.215.240.9. Redistribution subject to AIP license or copyright, see http://apl.aip.org/apl/copyright.jsp at constant rf. Thus, a varying $\phi_{H}$ complicates the interpretation of the experimental MRFM spectra since the spatial variation of both the field strength and $\phi_{H}$ must be known in order to evaluate the microscopic magnetic properties of the film.

Rather than a coil (used in previous FMR experiments ${ }^{3}$ ), we used a microstrip resonator with a characteristic frequency near $8 \mathrm{GHz}$ to provide the rf field. This increased the resonant field (applied in the film plane), $H_{\text {res }}$ $=\sqrt{\left(2 \pi M_{s}\right)^{2}+(\omega / \gamma)^{2}}-2 \pi M_{s} \simeq(\omega / \gamma)^{2} / 4 \pi M_{s}$, to a value sufficient to saturate the Co film $(\sim 100 \mathrm{G})$. Unlike other microstrip designs in which the resonator is located at the end of the transmission line, ${ }^{10,11}$ the resonator (see inset, Fig. 1 ) is located beside the transmission line. This provides good coupling between the resonator and the incoming transmission line $(-20 \mathrm{~dB})$ even with a fairly large gap $(\sim 0.2 \mathrm{~mm})$.

Modulation of the $z$ component of the Co spin magnetization was accomplished by modulating the amplitudes of both the external field $B_{0}$ and the rf power at two distinct frequencies whose difference was set equal to $f_{c}$ (i.e., anharmonic modulation). ${ }^{3,12}$

Figure 1 shows MRFM spectra of the three Co single layer films. The spectra were taken by measuring the inphase oscillation amplitude of the cantilever while sweeping the external magnetic field; increasing the applied field causes the position of the sensitive slice to move away from the source of the field gradient. When the sensitive slice intercepts the Co thin film, an increase in the cantilever vibration amplitude is observed. As in the YIG experiment, ${ }^{3}$ the MRFM signals from the Co films were so large that all spectra were taken at ambient pressure in order to reduce the $Q$ factor, and thus the response, of the cantilever ( $Q$ $\sim 15000$ in vacuum; $\sim 40$ in air). Because the signal-tonoise ratio is proportional to $\sqrt{Q}$, this result indicates that, in vacuum, the MRFM will be easily capable of detecting the FMR signal from Co films with similar lateral extent, but as thin as 20-50 A. By measuring the frequency dependence of the resonant field as a function of the position $(x, z)$ of the sample with respect to the bar magnet, we distinguish the individual contributions of the electromagnet and the bar magnet to the total field at the film and determine the detailed spatial dependence of the $\hat{x}$ and $\hat{z}$ components of the field of the bar magnet. From these data the angle $\phi_{H}$ was calculated. We find that the field gradient $\partial B_{\text {total }} / \partial z$ due to the bar magnet at the Co sample is $\sim 0.15 \mathrm{G} / \mu \mathrm{m}$. For our Co film, which is $\sim 200 \mu \mathrm{m}$ long, this corresponds to a $30 \mathrm{G}$ field difference across the film. Because the resonance linewidth of the Co films (between 50 and $100 \mathrm{G}$ ) is greater than this, we are not able to distinguish resonance signals arising from different spatial locations in the microscopic Co film by means of the applied field gradient. The larger field gradient produced by reducing the diameter of the magnet will be necessary to improve the spatial resolution of the experiment.

Analysis of our FMR spectra enable us to determine magnetic properties of the microscopic ferromagnetic films; in particular, these spectra reveal the dependence of the magnetic anisotropy and the film quality on the deposition 
sputtered film was determined by analysis of the dependence of the resonance field on $\phi_{H}$. This angle is varied by displacing the Co film with respect to the bar magnet in either the $x$ or $z$ direction, thus changing the $x$ and $z$ components of the magnetic field applied to the film. This result is shown in Fig. 2. The solid curve in Fig. 2 is a theoretical prediction for this dependence from classical FMR theory assuming only a demagnetization field. In particular, no crystalline anisotropy is observed; this absence is an expected consequence of the polycrystalline nature of the film. As shown, excellent agreement was obtained.

Figure 1 shows the FMR spectra of the three films taken at constant angle $\phi_{H} \simeq 33^{\circ}$. The resonant field position is larger in evaporated films indicating an additional anisotropy in these films. In particular, the effective demagnetization field, $4 \pi M_{\text {eff }}=4 \pi M_{s}-2 K_{u} / M_{s}$ is different (where $K_{u}$ is the uniaxial anisotropy energy density perpendicular to the film plane). This variation in $K_{u}$ could arise from different residual stresses developed during the film deposition. Assuming that the saturation magnetization $M_{s}$ for each of the Co films is the same as for bulk Co $\left(\sim 1400 \mathrm{emu} / \mathrm{cm}^{3}\right)$, we find that $2 K_{u} / M_{s} \sim 0$ for the sputtered film, $\sim 2.8 \mathrm{kG}$ for the $1000 \AA$ evaporated film, and $\sim 4.9 \mathrm{kG}$ for the $500 \AA$ evaporated film. These results indicate that the evaporated samples develop larger stress than the sputtered sample, and that the stress decreases with increasing film thickness.

A dependence of sample homogeneity on the deposition process is also evident in Fig. 1 from the variation of the FMR linewidth. The sputtered sample has a narrowest linewidth, about $45 \mathrm{G}$. The applied field gradient cannot explain this linewidth variation because the sputtered sample has the largest spatial extent along the $z$ axis (the direction along which the field gradient is largest). Thus, the linewidth reflects the quality or homogeneity of the Co film itself, and these measurements indicate that sputtering produces a more homogeneous film than does thermal evaporation. Between the two evaporated samples, the $500 \AA$ sample has the narrower resonance linewidth, possibly because a two stage evaporation was required for the $1000 \AA$ film, or possibly indicating that the film quality degrades as it becomes thicker.

In conclusion, a MRFM has been successfully used to detect FMR signals from microscopic Co single layer thin films with unprecedented sensitivity. These signals were obtained from a perpendicular-geometry MRFM operating at a frequency of $8 \mathrm{GHz}$. These conditions ensured that for modest resonance fields the applied field component in the plane of the film was sufficient to saturate the film. The large signal intensity indicates that the sensitivity of the current MRFM is adequate to detect FMR signals from microscopic metallic ferromagnets as thin as $20 \AA$. In fact, this has been verified in our most recent experiments on $50 \AA$ Co films. ${ }^{13}$ Although the field gradient in the present instrument is not large enough to distinguish FMR signals from different spatial locations in the film, our results demonstrate that MRFM detection of FMR has the sensitivity to enable microscopic studies of systems composed of thin film metallic ferromagnets. We have demonstrated, in particular, the ability to observe variations in magnetic anisotropy energy and film quality from one microscopic sample to another. In order to improve the spatial resolution, larger field gradients from smaller bar magnets are needed. Placing the magnetic probe on the cantilever is an essential step to enable experiments on samples prepared on well-characterized substrates. These improvements are presently underway.

The authors gratefully acknowledge fruitful discussions with Philip Wigen at Ohio State University and the support of the Center for Nonlinear Studies at Los Alamos National Laboratory. Work at Los Alamos was supported by the U.S. Department of Energy, Office of Basic Energy Sciences.

${ }^{1}$ P. E. Wigen, in Magnetic Multilayers, edited by L. H. Bennett and R. E. Watson (World Scientific, Singapore, 1994), pp. 183-226.

${ }^{2}$ B. Heinrich, in Ultrathin Magnetic Structures II, edited by B. Heinrich and J. A. C. Bland (Springer, Berlin, 1994), pp. 195-222.

${ }^{3}$ Z. Zhang, P. C. Hammel, and P. E. Wigen, Appl. Phys. Lett. 68, 2005 (1996).

${ }^{4}$ D. Rugar, C. S. Yannoni, and J. A. Sidles, Nature (London) 360, 563 (1992).

${ }^{5}$ D. Rugar, O. Züger, S. T. Hoen, C. S. Yannoni, H.-M. Vieth, and R. D. Kendrick, Science 264, 1560 (1994).

${ }^{6}$ J. A. Sidles, Appl. Phys. Lett. 58, 2854 (1991).

${ }^{7}$ J. A. Sidles, Phys. Rev. Lett. 68, 1124 (1992).

${ }^{8}$ O. Züger and D. Rugar, J. Appl. Phys. 75, 6211 (1994).

${ }^{9}$ P. C. Hammel, Z. Zhang, G. J. Moore, and M. L. Roukes, J. Low Temp. Phys. 101, 59 (1995).

${ }^{10}$ W. J. Wallace and R. H. Silsbee, Rev. Sci. Instrum. 62, 1754 (1991).

${ }^{11}$ K. Wago, O. Züger, J. Wegener, R. Kendrick, C. S. Yannoni, and D. Rugar, Rev. Sci. Instrum. 68, 1823 (1997).

${ }^{12}$ K. J. Bruland, J. Krzystek, J. L. Garbini, and J. A. Sidles, Rev. Sci. Instrum. 66, 2853 (1995).

${ }^{13}$ M. Midzor, M. L. Roukes, B. J. Suh, Z. Zhang, P. C. Hammel, and J. R. Childress (unpublished). 